\title{
Factors Associated with Acceptability of HIV Self-Testing Among Health Care Workers in Kenya
}

\author{
Samuel Kalibala $\cdot$ Waimar Tun $\cdot$ Peter Cherutich $\cdot$ \\ Anne Nganga $\cdot$ Erick Oweya $\cdot$ Patricia Oluoch
}

Published online: 29 June 2014

(c) Springer Science+Business Media New York 2014

\begin{abstract}
Health care workers (HCWs) in sub-Saharan Africa are at a high risk of HIV infection from both sexual and occupational exposures. However, many do not seek HIV testing. This paper examines the acceptability of an unsupervised facility-based HIV self-testing (HIVST) intervention among HCWs and their partners and factors associated with uptake of HIVST among HCWs. HCWs in seven large Kenyan hospitals were invited to participate in pre-HIVST information sessions during which they were offered HIVST kits to take home for selftesting. A post-intervention survey was conducted among 765 HCWs. Forty-one percent attended the information session; of those, $89 \%$ took the HIVST kits and of those, $85 \%$ self-tested. Thirty-four percent of surveyed HCWs used the HIVST to test themselves. Of those who took the HIVST kit and had partners, $73 \%$ gave the kit to their partner and $86 \%$ of them indicated their partner self-tested. Factors positively associated with use of the HIVST on self were being female, being single, and being a $\mathrm{HCW}$ from Homa Bay Hospital (located in a high HIV prevalence area). HIVST is acceptable to HCWs and their
\end{abstract}

\footnotetext{
S. Kalibala $(\bowtie) \cdot$ W. Tun

Population Council, HIV and AIDS Program, 4301 Connecticut

Avenue, Suite 280, Washington, DC 20008, USA

e-mail: skalibala@popcouncil.org

P. Cherutich - A. Nganga

National AIDS and STD Control Program (NASCOP), Ministry

of Health, Nairobi, Kenya

E. Oweya $\cdot$ P. Oluoch

Centers for Disease Control and Prevention, Nairobi, Kenya
}

partners. However, strategies are needed to increase HCWs attendance at pre-implementation information sessions.

Keywords HIV self-test · Health care workers · HIV testing

\section{Introduction}

Health care workers (HCWs) based in sub-Saharan Africa are at high risk of HIV infection, both from their sexual relations and from occupational exposure [1]. It is estimated that 2 million needle stick injuries (NSIs) occur among HCWs per year worldwide. The World Health Organization (WHO) estimates that $40 \%$ of the hepatitis B and $\mathrm{C}$ infections and $2.5 \%$ of the HIV infections among HCWs are attributable to NSIs [2]. In many sub-Saharan African countries, the number of available trained HCWs has historically been inadequate, and countries have suffered from scarcities of almost all cadres of HCWs, primarily due to morbidity and mortality as a result of HIV and AIDS [3].

The high rates of AIDS-related deaths among HCWs reflect low uptake of HIV services among this group [4]. Despite the risk of HIV infection among HCWs and the availability of antiretroviral drugs (ARVs), many HCWs are reluctant to seek HIV testing, and therefore, do not access HIV treatment and prevention services [4]. Additionally, HIV sero-conversion after NSI could be prevented using ARVs, generally referred to as post-exposure prophylaxis (PEP). According to the Kenyan Ministry of Health guidelines, an HIV test and diagnosis is required before PEP can be initiated [5]. In a study among HCWs in central Kenya who had had NSIs, the uptake of PEP was only $4 \%$ [6]. Such low uptake was primarily due to HCWs 
fear of HIV testing and their perception of NSIs as low risk [7]. Similarly, the Kenya Health Workers Survey also revealed that HCWs had anxieties about testing for HIV fearing others would know their HIV status, fearing a lack of privacy, and experiencing stigma from their colleagues who may assume they are HIV positive [8].

Globally, there is increasing evidence that HIV self-testing (HIVST) is commonly practiced among HCWs [4]. In Ethiopia, a study among HCWs showed that $70 \%$ of those who had ever had an HIV test had also self-tested [9], the main reason being the need for privacy. Thus, a wellimplemented HIVST program could be an effective way for increasing uptake of HIV testing with increased privacy, and increasing entry into HIV prevention, care and treatment services, such as early ARVs and access to PEP. Additionally, HIVST could also be at a lower cost and require fewer human resources than alternative approaches [3].

Providing HIVST for HCWs would increase access to knowledge of HIV status in an atmosphere that is private, not threatening, and devoid of anxiety about stigma from colleagues and other clients. The Kenya Health Workers Survey revealed that HCWs have a desire $(73 \%)$ to have a self-test for HIV [8]. A study across five African countries found self-testing to be acceptable to HCWs; they also viewed self-testing as an opportunity to increase family testing [4]. A meeting of experts on HIVST convened by WHO and UNAIDS in Geneva in April 2013 issued a consensus statement highlighting that self-testing kits are already being used for HIVST in various settings, and may be particularly useful for HCWs in high prevalence settings [10].

We conducted a study to examine the implementation of an unsupervised facility-based HIVST intervention among HCWs in seven hospitals in Kenya. The primary objective of the study was to assess the acceptability of HIVST by measuring the proportion of HCWs that would conduct self-testing if availed HIVST kits and instructed on their use. The secondary objective was to examine factors associated with HIVST uptake.

\section{Methods}

A cross-sectional survey was conducted among HCWs in provincial and district hospitals after the implementation of the HIVST intervention between December 2009 and February 2010. A summary of the methods is described here; full details have been previously provided [11].

Hospitals were randomly selected from each region of Kenya. Four of the hospitals were urban: Bungoma (Western Region), Malindi (Coast Region), Mbagathi (Nairobi Region), and Makueni (Eastern Region). The remaining three were rural: Homa Bay (Nyanza Region),
Nanyuki (Central Region), and Garissa (North Eastern Region). The intervention and survey were limited to clinical personnel in the hospitals, namely doctors, nurses, clinical officers, laboratory technicians, social workers, and counselors. The intervention was implemented by a trained team from Crystal Hill Limited, a local consultancy organization. The intervention team requested each hospital director to select two HCWs with the skills to provide HIV testing and counseling (HTC) services, who would function as the on-site intervention coordinators. The research component was implemented directly by a study team from the Population Council, an international non-government organization.

The intervention team visited each hospital on the appointed day and invited all eligible $\mathrm{HCW}$ s who were interested in self-testing for HIV to participate in information sessions. The sessions included information on the HIV epidemic in Kenya, HIV risks, available HIV testing options for HCWs, and instructions on the appropriate use of the test kits (CalypteR Aware ${ }^{\mathrm{TM}}$ oral HIV test), including a live demonstration and a video show on its use. In total, three to four sessions were conducted over the twoday period at each hospital. At the end of the pre-HIVST information session, HCWs were offered HIVST kits. Participants who wanted an HIVST kit were given at least two test kits. Participants were also encouraged to offer their partner or spouse the kit and teach them how to use it.

In addition to the information session, participants also received detailed written information, leaflets created by the intervention team, contact information of local HTC and ARV facilities, telephone numbers for the HIVST intervention hotline and a test kit containing written instructions for use provided by the manufacturer. All materials were in the English language. At each hospital, HCWs who did not attend a pre-HIVST session were informed that they could access test kits and additional information on HIVST from the on-site coordinators.

The HIVST intervention team and the on-site coordinators were available for 1 month after the information sessions to provide support and referrals to any HCW through a telephone hotline. The intervention team maintained regular telephone contact with the on-site coordinators and provided additional kits and materials as necessary to the study sites and ensured that there were no stock-outs of the HIVST kits during the one-month period of the intervention. A mobile phone company was contracted to provide the toll-free services of a telephone hotline, and the on-site coordinators were provided a log for recording the phone calls received on the hotline.

The study team conducted a structured survey of HCWs in all seven hospitals a month after the HIVST kits were distributed to document uptake and usage as well as assess the acceptability of the HIVST intervention. Survey 
participants were recruited through a convenience sample and included HCWs who did and did not attend the preHIVST session, as well as HCWs who did and did not take an HIVST kit. Respondents were asked about their attendance at the pre-HIVST information session, uptake of HIVST kits, use of the test kit (by oneself and their partner(s)) and their perceptions about HIVST kits. Each survey lasted less than $30 \mathrm{~min}$. At no point in the survey were the study participants asked to reveal their HIV status.

\section{Analysis}

To measure acceptability of HIVST, we calculated the proportion of HCWs that reported attending the pre-HST information session, the proportion that reported taking the HIVST kits, and the proportion that reported use of the HIVST (by the HCW). To assess factors associated with HIVST uptake, we compared the demographic and behavioral characteristics of HCWs by attendance at preHIVST session and self-reported use of the HIVST kit using Chi squared test for categorical variables and the Mann-Whitney $U$ Test for continuous variables. All $p$ values were two-sided. Multivariate logistic regression was used to examine factors associated with self-reported use of HIVST kit on self. Factors associated with the outcome in the univariate analyses at the $p=0.10$ level were included in the multivariate model, as well as age. Analyses were all conducted using SAS 9.4 (SAS Institute, Cary, NC, USA).

The study protocol was approved by the ethical review boards of the Population Council, the US Centers for Disease Control and Prevention (CDC), and the Kenyatta National Hospital. All participants provided verbal and/or written consent. The HIV self-test kits were donated by Calypte Biomedical Corporation. The study was funded by the U.S. Centers for Disease Control and Prevention. The authors have declared no conflict of interest.

\section{Results}

Service Uptake Data from HIVST Service Delivery and Session Attendance Logs

A total of $842 \mathrm{HCW}$ attended the pre-HIVST information sessions. Of those, 820/842 (97\%) took at least one test kit. The majority of attendees (629/820; $77 \%$ ) received the test kits immediately after the information session. The remainder $(191 / 820 ; 23 \%)$ received the kits from the on-site team after the sessions. On-site coordinators indicated that there was only one telephone call to the hotline. The call was regarding the test procedure and did not involve counseling. Hence, the telephone logs were not analyzed.
Post-intervention Survey

A total of $765 \mathrm{HCW}$ responded to the post-intervention survey and $313(41 \%)$ had attended the pre-HIVST information sessions (Fig. 1). Of those who attended a session, the majority $(89 \% ; 278 / 313)$ took at least one HIVST kit, and $85 \%(237 / 278)$ of them tested themselves with the HIVST kits. A small percentage of respondents (7\%; 33/452) who did not attend a session reported that they took a HIVST kit, of which $79 \%$ (26/33) self-tested using the provided kit. However, the majority of respondents who did not attend the pre-HIVST information sessions did not take test kits or test himsef or herself. Ultimately, a total of $34 \%(263 / 765)$ of surveyed HCWs tested themselves.

Table 1 describes the characteristics of the survey respondents. The median age was 33, two-thirds (67\%) were female, $59 \%$ were married or cohabiting, and the majority were registered nurses $(32 \%)$, enrolled nurses (24\%) or clinical officers (24\%). Approximately onethird $(30 \%)$ reported not having used a condom at their last sexual act. HIV testing prior to the HIVST intervention was high with $92 \%$ ever having tested, and of those, $26 \%$ indicated they self-tested for their last HIV test prior to the HIVST intervention.

Table 1 compares the characteristics of those who attended the pre-HIVST information sessions and those who did not. Female HCWs were significantly more likely than male HCWs to attend the pre-HIVST information sessions $(p<0.01)$. There was also a difference by professional cadre; clinical officers and HTC counselors were significantly more likely to attend and lab technicians were less likely to attend ( $p<0.0001)$. There was a significant difference in attendance by hospital and region of the country. Those from Homa Bay Hospital (in western Kenya where HIV prevalence is high) were more likely to attend the sessions and those at Garissa Hospital (in northern Kenya where HIV prevalence is low) were less likely $(p<0.0001)$. There also was a barely significant difference in attendance by condom use $(p=0.053)$. Those who used a condom at last sex were more likely to attend than those who did not. There was no statistically significant difference in pre-HIVST information session attendance by age, previous HIV testing, and time of last HIV test.

Of the survey respondents that reported taking an HIVST kit, $49 \%$ (150/311) took five or more HIVST kits. However, respondents who did not attend the pre-HIVST session were significantly more likely to take only one HIVST kit compared to those who attended the session (39 vs. $6 \% ; p<0.001)$. Among those who took the HIVST kit, 175 had partners, and of these 127 (73\%) indicated that their partner took the HIVST kit. Of those who gave a 
Fig. 1 Cascade of healthcare workers who attended the preHIVST session, took HIVST kits, and used the HIVST kit on self among healthcare workers who participated in the survey $(\mathrm{N}=765)$

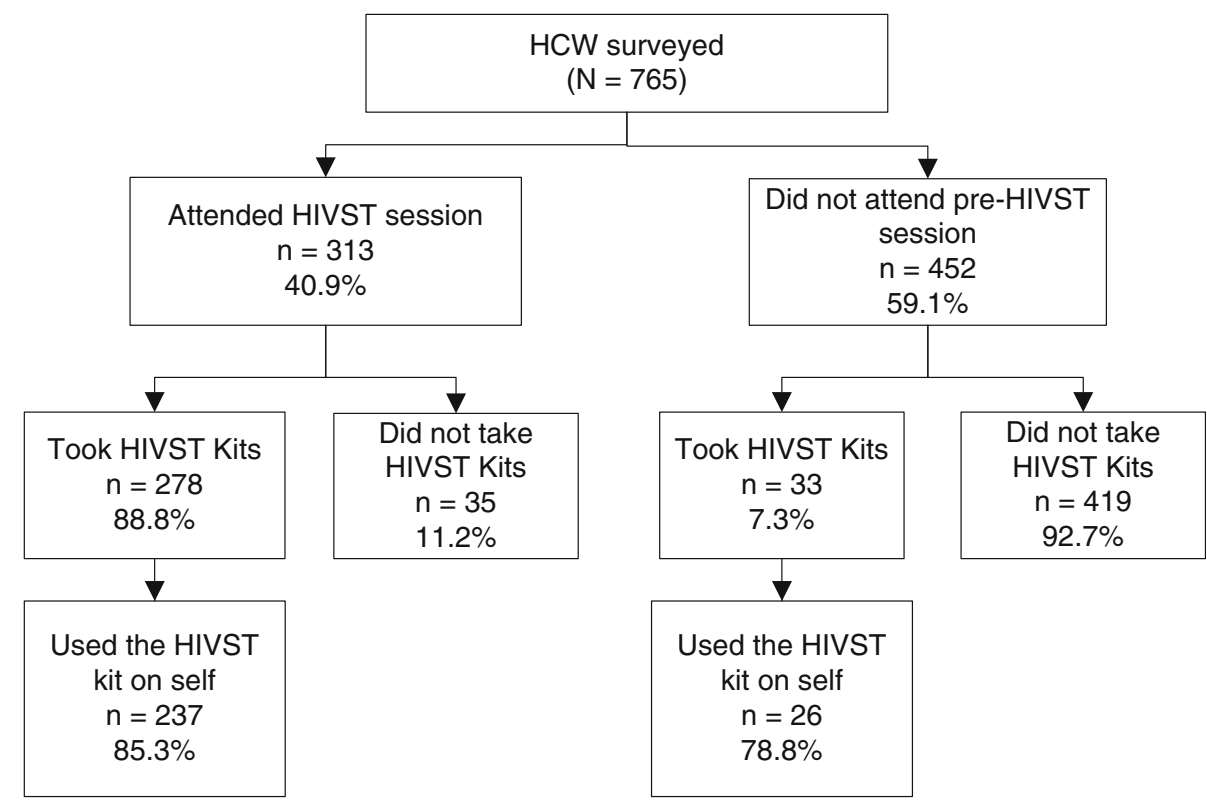

HIVST kit to a partner, $86 \%(108 / 126)$ reported that the partner tested themselves with the HIVST kit. There was no significant difference, regarding the proportion whose partner took the HIVST kit and partner self-testing, by whether the participant attended the pre-HIVST information session.

All survey respondents were asked about their perceptions about self-testing for HIV. The majority (89\%) indicated that they would recommend HIVST to fellow HCWs, and those who attended the pre-HIVST information session were more likely to recommend HIVST compared to those who had not attended (93 vs. $86 \% ; p<0.01$ ) (Table 1). Additionally, half indicated that HIVST could be abused by HCWs, mainly through the testing of a partner without their consent $(44 \%)$. There was no significant difference between those who attended the information session and those who did not (Table 1).

Table 2 shows the factors associated with self-reported HIVST use. In multivariate analysis, females were significantly more likely to use the HIVST kit compared to males (adjusted odd ratio (AOR): 1.7; $95 \% \mathrm{CI}: 1.2-2.3$ ) and those who are married or living with a partner were significantly less likely to use the HIVST test kit compared to those who are single (AOR: 0.7; $95 \%$ CI: 0.4-1.0). There was a significant difference in the use of the HIVST test kit by site; HCWs from Garissa were significantly less likely to test themselves with HIVST compared to those at Bungoma (AOR: 0.5; $95 \%$ CI: 0.2-0.8) and although not significant, those from Homa Bay were slightly more likely to test themselves with HIVST than those from Bungoma (AOR: 1.6; $95 \%$ CI: 0.9-2.8). The communities around Bungoma and Homa Bay have high HIV prevalence; Garissa has low HIV prevalence [16]. There was no significant difference in the use of HIVST test by age, professional cadre, previous HIV testing, and sexual behavior.

The majority of survey respondents who reported using an HIVST kit indicated that the HIVST kit was 'very easy' to use (94\%) and that the instructions were 'very easy' to understand (95\%) (Data not shown). HIVST users also indicated that they discussed the HIVST experience with their sex partner $(55 \%)$, colleague $(34 \%)$, and friend (21\%); however, $18 \%$ indicated they did not discuss it with anyone. Only one individual among these respondents reported calling the telephone hotline to discuss HIV test results.

\section{Discussion}

According to the consensus statement from the first international symposium on HIVST [10], despite limited experience and evidence, HIVST has a vast potential to scale-up access to HTC services. However, before promoting it widely, the WHO/UNAIDS meeting highlighted the need for implementation science research to contribute to the evidence base for HIVST programming. It was further stated that before international guidance for national programs can be developed, more research is needed to provide the evidence that HIVST is a viable option at the national level. Our study is among the few that contribute to this evidence-base by giving data on acceptability as well as the demographic and behavioral factors that are associated with the uptake of HIVST among HCWs in Kenya. While this study was conducted in seven hospitals in one country, and therefore cannot be generalized, we 
Table 1 Socio-demographic and other key characteristics of healthcare workers who completed the survey $(\mathrm{N}=765)$

\begin{tabular}{|c|c|c|c|c|}
\hline & $\begin{array}{l}\text { Total } \\
(\mathrm{N}=765) \\
\%(\mathrm{n})\end{array}$ & $\begin{array}{l}\text { Attended HIVST } \\
\text { Session }(\mathrm{n}=313) \\
\%(\mathrm{n})\end{array}$ & $\begin{array}{l}\text { Did not attend } \\
\text { HIVST session } \\
(n=452) \%(n)\end{array}$ & $p$ value $^{\mathrm{a}}$ \\
\hline Median age (IQR) & $33(18)$ & $32(20)$ & $33(17)$ & $0.751^{\mathrm{b}}$ \\
\hline \multicolumn{5}{|l|}{ Sex } \\
\hline Male & $33.5(256)$ & $28.1(88)$ & $37.2(168)$ & \multirow[t]{2}{*}{$<0.01$} \\
\hline Female & $66.5(509)$ & $71.9(225)$ & $62.8(284)$ & \\
\hline \multicolumn{5}{|l|}{ Professional cadre } \\
\hline Registered nurse & $31.8(243)$ & $31.6(99)$ & $31.9(144)$ & \multirow[t]{7}{*}{$<0.0001$} \\
\hline Enrolled nurse & $23.8(182)$ & $21.4(67)$ & $25.4(115)$ & \\
\hline Clinical officer & $23.9(183)$ & $28.1(88)$ & $21.0(95)$ & \\
\hline Lab technician & $9.5(73)$ & $4.8(15)$ & $12.8(58)$ & \\
\hline HTC counselor & $4.6(35)$ & $8.3(26)$ & $2.0(9)$ & \\
\hline Medical doctor & $4.2(32)$ & $3.5(11)$ & $4.7(21)$ & \\
\hline Social worker & $2.22(17)$ & $2.24(7)$ & $2.21(10)$ & \\
\hline \multicolumn{5}{|l|}{ Marital status } \\
\hline Single (Never married) & $35.7(273)$ & $38.7(121)$ & $33.6(152)$ & \multirow[t]{4}{*}{0.3465} \\
\hline Married or living with partner & $59.4(454)$ & $56.9(178)$ & $61.1(276)$ & \\
\hline Divorced/widowed/separated & $5.0(38)$ & $4.5(14)$ & $5.3(24)$ & \\
\hline Site & $(\mathrm{N}=765)$ & $(\mathrm{N}=313)$ & $(\mathrm{N}=452)$ & \\
\hline Homa Bay & $14.4(110)$ & $19.5(61)$ & $10.8(49)$ & \multirow[t]{7}{*}{$<0.0001$} \\
\hline Bungoma & $14.3(109)$ & $16.0(50)$ & $13.1(59)$ & \\
\hline Makueni & $11.4(87)$ & $11.5(36)$ & $11.3(51)$ & \\
\hline Nanyuki & $10.5(80)$ & $11.2(35)$ & $10.0(45)$ & \\
\hline Malindi & $12.6(96)$ & $15.7(49)$ & $10.4(47)$ & \\
\hline Mbagathi & $22.4(171)$ & $19.2(60)$ & $24.6(111)$ & \\
\hline Garissa & $14.6(112)$ & $7.0(22)$ & $20.0(90)$ & \\
\hline \multicolumn{5}{|l|}{ HIV testing prior to the HIVST intervention } \\
\hline Yes & $92.0(704)$ & $93.1(292)$ & $91.2(412)$ & \multirow[t]{3}{*}{0.283} \\
\hline No & $8.0(61)$ & $6.7(21)$ & $8.9(40)$ & \\
\hline Time of last HIV test before HIVST campaign & $(\mathrm{N}=704)$ & $(\mathrm{N}=292)$ & $(\mathrm{N}=412)$ & \\
\hline Less than 12 months ago & $76.3(537)$ & $77.1(225)$ & $75.7(312)$ & \multirow[t]{4}{*}{0.907} \\
\hline $12-23$ months ago & $8.7(61)$ & $8.6(25)$ & $8.7(36)$ & \\
\hline 2 or more years ago & $15.1(106)$ & $14.3(42)$ & $15.5(64)$ & \\
\hline $\begin{array}{l}\text { How the last test results were received } \\
\text { (among those tested previously) }\end{array}$ & $(\mathrm{N}=690)$ & $(\mathrm{N}=284)$ & $(\mathrm{N}=406)$ & \\
\hline Counseled by counselor & $44.6(308)$ & $44.4(126)$ & $44.8(182)$ & \multirow[t]{4}{*}{0.273} \\
\hline Given results without counseling & $29.0(200)$ & $26.4(75)$ & $30.8(125)$ & \\
\hline Self-tested & $26.4(182)$ & $29.2(83)$ & $24.4(99)$ & \\
\hline Sexual activity ${ }^{\mathrm{c}}$ & $(\mathrm{N}=709)$ & $(\mathrm{N}=290)$ & $(\mathrm{N}=419)$ & \\
\hline Had sex in last 12 months with regular partner & $85.9(609)$ & $83.8(243)$ & $87.4(366)$ & \multirow[t]{4}{*}{0.326} \\
\hline Had sex in last 12 months with a non-regular partner & $1.3(13)$ & $1.7(5)$ & $1.9(8)$ & \\
\hline No (did not have sex in the last 12 months) & $12.3(87)$ & $14.5(42)$ & $10.7(45)$ & \\
\hline Condom use at last sex & $(\mathrm{N}=713)$ & $(\mathrm{N}=291)$ & $(\mathrm{N}=422)$ & \\
\hline Had sex without condom & $30.0(214)$ & $34.0(99)$ & $27.3(115)$ & \multirow[t]{3}{*}{0.053} \\
\hline Had sex with condom & $70.0(499)$ & $66.0(192)$ & $72.8(307)$ & \\
\hline Took the HIVST kit & $(\mathrm{N}=765)$ & $(\mathrm{N}=313)$ & $(\mathrm{N}=452)$ & \\
\hline Yes & $40.7(311)$ & $88.8(278)$ & $7.3(33)$ & \multirow[t]{3}{*}{$<0.0001$} \\
\hline No & $59.3(454)$ & $11.2(35)$ & $92.7(419)$ & \\
\hline Number of HIVST kits taken & $(\mathrm{N}=308)$ & $(\mathrm{N}=275)$ & $(\mathrm{N}=33)$ & \\
\hline
\end{tabular}


Table 1 continued

\begin{tabular}{|c|c|c|c|c|}
\hline & $\begin{array}{l}\text { Total } \\
(\mathrm{N}=765) \\
\%(\mathrm{n})\end{array}$ & $\begin{array}{l}\text { Attended HIVST } \\
\text { Session }(\mathrm{n}=313) \\
\%(\mathrm{n})\end{array}$ & $\begin{array}{l}\text { Did not attend } \\
\text { HIVST session } \\
(\mathrm{n}=452) \%(\mathrm{n})\end{array}$ & $p$ value $^{\mathrm{a}}$ \\
\hline One & $9.7(30)$ & $6.2(17)$ & $39.4(13)$ & $<0.001$ \\
\hline Two & $41.6(128)$ & $41.5(114)$ & $42.4(14)$ & \\
\hline 5 or more & $48.7(150)$ & $52.4(144)$ & $18.2(6)$ & \\
\hline Tested themselves with HIVST kit & $(\mathrm{N}=765)$ & $(\mathrm{N}=313)$ & $(\mathrm{N}=452)$ & \\
\hline Yes & $34.4(263)$ & 75.7 (237) & $5.8(26)$ & $<0.0001$ \\
\hline No & $65.6(502)$ & $24.3(76)$ & $94.2(426)$ & \\
\hline $\begin{array}{l}\text { Partner took the HIVST kit among those who took HIVST kit } \\
\text { and have a partner }\end{array}$ & $(\mathrm{N}=175)$ & $(\mathrm{N}=156)$ & $(\mathrm{N}=19)$ & \\
\hline Yes & $72.5(127)$ & $72.4(113)$ & $68.4(13)$ & 0.863 \\
\hline No & $27.4(48)$ & $27.5(43)$ & $31.6(6)$ & \\
\hline Partner tested with HIVST kit & $(\mathrm{N}=126)$ & $(\mathrm{N}=113)$ & $(\mathrm{N}=13)$ & \\
\hline Yes & $85.7(108)$ & $85.0(96)$ & $92.3(12)$ & 0.728 \\
\hline No & $14.3(18)$ & $15.0(17)$ & $7.7(1)$ & \\
\hline Would recommend HIVST to fellow HCWs & $(\mathrm{N}=765)$ & $(\mathrm{N}=313)$ & $(\mathrm{N}=452)$ & \\
\hline Yes & $88.9(680)$ & $93.0(291)$ & $86.1(389)$ & $<0.01$ \\
\hline No & $11.1(85)$ & $7.0(22)$ & $13.9(63)$ & \\
\hline Thinks HIVST can be abused by HCW & $(\mathrm{N}=764)$ & $(\mathrm{N}=313)$ & $(\mathrm{N}=451)$ & \\
\hline Yes & $49.9(381)$ & $50.2(157)$ & $49.7(224)$ & 0.8935 \\
\hline No or Don't Know & $50.1(383)$ & $49.8(156)$ & $50.3(227)$ & \\
\hline Ways in which HIVST can be abused by HCWs & $(\mathrm{N}=381)$ & $(\mathrm{N}=157)$ & $(\mathrm{N}=224)$ & \\
\hline \multicolumn{5}{|l|}{ Testing a partner without their consent } \\
\hline Yes & $44.4(169)$ & $43.9(69)$ & $44.6(100)$ & 0.8933 \\
\hline No & $55.6(212)$ & $56.1(88)$ & $55.4(124)$ & \\
\hline \multicolumn{5}{|l|}{ Testing children/infants } \\
\hline Yes & $12.1(46)$ & $12.7(20)$ & $11.6(26)$ & 0.7386 \\
\hline No & $87.9(335)$ & $87.3(137)$ & $88.4(198)$ & \\
\hline \multicolumn{5}{|c|}{ Infecting partner/others by injecting them with their infected blood if found positive } \\
\hline Yes & $7.9(30)$ & $8.9(14)$ & $7.1(16)$ & 0.5268 \\
\hline No & $92.1(351)$ & $91.1(143)$ & $92.9(208)$ & \\
\hline \multicolumn{5}{|l|}{ Selling of test kit } \\
\hline Yes & $21.0(80)$ & $24.2(38)$ & $18.8(42)$ & 0.1983 \\
\hline No & $79.0(301)$ & $75.8(119)$ & $81.2(182)$ & \\
\hline \multicolumn{5}{|l|}{ Non-usage of test kit (no feedback) } \\
\hline Yes & $5.0(19)$ & $7.0(11)$ & $3.6(8)$ & 0.1295 \\
\hline No & $95.0(362)$ & $93.0(146)$ & $96.4(216)$ & \\
\hline
\end{tabular}

$I Q R$ Interquartile range

${ }^{a}$ Chi squared test unless specified

b Mann-Whitney U Test

c A regular partner was defined as a spouse, boyfriend, girlfriend, or cohabiting partner; a non-regular partner was a casual partner, someone who respondent just met or a commercial partner (someone with whom the participant had sex in exchange for money, goods or services)

d This question was only asked to those who were married or living with a partner

believe that this information can inform further research on HIVST models targeting HCWs in Africa.

This paper has shown a high acceptability of HIVST among HCWs in Kenya and has examined factors associated with the uptake of this service in this population.
There was a high frequency of HIV testing (92\%) among HCWs prior to the HIVST intervention, suggesting that this is a population who generally test for HIV regularly. HCWs perceived themselves at risk for both occupational and sexual transmission and knew the importance of 
Table 2 Factors associated with using the HIV self-test kit among health care workers $(\mathrm{N}=765)$

\begin{tabular}{|c|c|c|c|c|c|c|c|}
\hline & $\begin{array}{l}\text { Self-tested with } \\
\text { HIVST } N=263 \\
\%(\mathrm{n})\end{array}$ & $\begin{array}{l}\text { Did not self-test with } \\
\text { HIVST N }=502 \\
\%(\mathrm{n})\end{array}$ & $p$-value ${ }^{\mathrm{a}}$ & Odds ratio & $p$-value ${ }^{\mathrm{a}}$ & $\begin{array}{l}\text { Adjusted } \\
\text { odds ratio }\end{array}$ & $p$-value \\
\hline \multicolumn{8}{|l|}{ Age } \\
\hline$\geq 33$ years & $46.0(121)$ & $52.4(263)$ & 0.094 & $1.3(1.0-1.7)$ & 0.0938 & $1.2(0.8-1.8)$ & 0.3264 \\
\hline$<33$ years & $54.0(142)$ & $47.6(239)$ & & & & & \\
\hline \multicolumn{8}{|l|}{ Sex } \\
\hline Male & $28.5(75)$ & $36.1(181)$ & $<0.05$ & $1.4(1.0-2.0)$ & $<0.01$ & $1.7(1.2-2.3)$ & $<0.01$ \\
\hline Female & $71.5(188)$ & $63.9(321)$ & & & & & \\
\hline \multicolumn{8}{|l|}{ Professional cadre } \\
\hline Registered nurse & $31.6(83)$ & $31.9(160)$ & 0.365 & & 0.3665 & & \\
\hline Clinical officer/Medical doctor & $31.2(82)$ & $26.5(133)$ & & $1.2(0.8-1.7)$ & & & \\
\hline Enrolled nurse & $20.5(54)$ & $25.5(128)$ & & $0.8(0.5-1.2)$ & & & \\
\hline Other & $16.7(44)$ & $16.1(81)$ & & $1.0(0.7-1.6)$ & & & \\
\hline \multicolumn{8}{|l|}{ Marital status } \\
\hline Single & $41.1(108)$ & $32.9(165)$ & 0.075 & & 0.0762 & & \\
\hline Married or living with partner & $54.8(144)$ & $61.8(310)$ & & $0.7(0.5-1.0)$ & & $0.7(0.4-1.0)$ & $<0.05$ \\
\hline Divorced/widowed/separated & $4.2(11)$ & $5.4(27)$ & & $0.6(0.3-1.3)$ & & $0.5(0.2-1.0)$ & 0.0641 \\
\hline \multicolumn{8}{|l|}{ Site } \\
\hline Bungoma & $14.8(39)$ & $13.9(70)$ & $<0.01$ & & $<0.01$ & & \\
\hline Homa Bay & $19.0(50)$ & $12.0(60)$ & & $1.5(0.9-2.6)$ & & $1.6(0.9-2.8)$ & 0.0985 \\
\hline Makueni & $10.3(27)$ & $12.0(60)$ & & $0.8(0.4-1.5)$ & & $0.9(0.5-1.7)$ & 0.7643 \\
\hline Nanyuki & $12.9(34)$ & $9.2(46)$ & & $1.3(0.7-2.4)$ & & $1.4(0.8-2.5)$ & 0.2760 \\
\hline Malindi & $14.8(39)$ & $11.4(57)$ & & $1.2(0.7-2.2)$ & & $1.2(0.7-2.2)$ & 0.5018 \\
\hline Mbagathi & $18.6(49)$ & $24.3(122)$ & & $0.7(0.4-1.2)$ & & $0.6(0.4-1.1)$ & 0.0884 \\
\hline Garissa & $9.5(25)$ & $17.3(87)$ & & $0.5(0.3-0.9)$ & & $0.5(0.2-0.8)$ & $<0.05$ \\
\hline $\begin{array}{l}\text { How the last test results were received } \\
\text { (among those tested previously) }(\mathrm{N}=690)\end{array}$ & $(\mathrm{N}=241)$ & $(\mathrm{N}=449)$ & & & 0.2154 & & \\
\hline Counseled by counselor & $44.4(107)$ & $44.8(201)$ & 0.214 & & & & \\
\hline Given results without counseling & $25.7(62)$ & $30.7(138)$ & & $0.8(0.6-1.2)$ & & & \\
\hline Self-tested & $29.9(72)$ & $24.5(110)$ & & $0.7(0.5-1.0)$ & & & \\
\hline Sexual activity $^{\mathrm{b}}(\mathrm{N}=709)$ & $(\mathrm{N}=238)$ & $(\mathrm{N}=471)$ & & & & & \\
\hline Had sex in last 12 months with regular partner & $84.5(201)$ & $86.6(408)$ & 0.646 & & 0.6464 & & \\
\hline $\begin{array}{l}\text { Had sex in last } 12 \text { months with casual/ } \\
\text { commercial partner }\end{array}$ & $1.7(4)$ & $1.9(9)$ & & $0.9(0.3-3.0)$ & & & \\
\hline Did not have sex in the last 12 months & $13.9(33)$ & $11.5(54)$ & & $1.2(0.8-2.0)$ & & & \\
\hline Condom use at last sex $(\mathrm{N}=713)$ & $(\mathrm{N}=239)$ & $(\mathrm{N}=474)$ & & & & & \\
\hline Had sex without condom & $67.4(161)$ & $71.3(338)$ & 0.278 & & & & \\
\hline Had sex with condom & $32.6(78)$ & $28.7(136)$ & & $1.2(0.3-1.2)$ & 0.2783 & & \\
\hline
\end{tabular}

a Chi squared test

b A regular partner was defined as a spouse, boyfriend, girlfriend, or cohabiting partner; a non-regular partner was a casual partner, someone who respondent just met or a commercial partner (someone with whom the participant had sex in exchange for money, goods or services)

getting tested for HIV [6]. It is notable that one-quarter of survey respondents indicated that their last HIV test prior to the HIVST intervention was a self-test. This suggests that there was need for an HIV testing option that would allow them greater privacy. Therefore, consistent with this desire to self-test for HIV, when test kits were offered to HCWs in the seven participating hospitals, acceptance and use of the HIVST kits was high. Another key finding was that HCWs who did not participate in the intervention, although a small percentage, also did access and use the HIVST kits.

Our data suggests that there is a high frequency of HIV testing among these HCWs. It is possible that with roll-out of a HIVST program, the frequency of HIV testing among HCWs could increase. At the international symposium on HIVST [10], a concern was raised that frequent HIVST alone should not be used as a preventive strategy. Indeed, WHO has highlighted the importance that messaging about 
HIVST should clearly explain that a self-test does not provide a diagnosis and requires further testing, that HIVST should be provided with clear instructions for use and interpretation, as well as how to access HIV prevention, care and treatment services [11].

As highlighted in a recent mathematical model, men who have sex with men (MSM) in Seattle who replace facility-based HTC using antigen-antibody combination assays and nucleic acid amplification tests with an oral fluid-based HIVST kit could lead to an increase in the transmission of HIV among this population. Based on the models assumptions, this is primarily because of the lower titres of antibodies and the longer window period of the available oral HIVSTVST kit, which will result in more false negative results than facility-based HTC [12]. Therefore, it is important that the provision of HIVST, as with all HTC services, include programming and messaging about how to access and link to HIV prevention, care and treatment services.

A number of factors were associated with the uptake of HIVST among these HCWs. Professional cadre was associated with attending the pre-HIVST information session, but it was not associated with the self-reported use of HIVST. Thus, professional cadre may simply suggest who may have the time to attend the sessions. Factors that were positively associated with the use of an HIVST kit were being female and single, but survey respondents who were married or in a steady relationship were less likely to use an HIVST kit or participate in the information sessions. However, according to the Kenya Modes of Transmission Study, heterosexual transmission within regular or steady relationships accounts for $44 \%$ of all new HIV infections in Kenya [13]. Thus, it is crucial for future programs to promote HIVST to $\mathrm{HCW}$ who are married or in a steady relationship.

Being a HCW from Garissa Hospital, where HIV prevalence is low, was negatively associated with attending the pre-HIVST information session and use of the HIVST test kit while being a HCW from Homa Bay Hospital, where HIV prevalence is high, was positively associated with both these aspects of the program. Further research is required to examine how characteristics of a hospital or the HIV prevalence in the surrounding area may influence the use of HIVST by HCWs in that hospital. A review of the literature found only a few studies examining factors affecting the uptake of HIV testing among HCWs in Africa. A study in South Africa found no significant associations between HCWs who reported having undergone HTC in the previous year, and their age, gender and marital status [14]. In a study among HCWs in Burkina Faso laboratory workers, and HCWs those who had multiple partners were more likely to have tested for HIV [15]. It should be noted that these two studies were not examining HIVST but HIV testing in general. However, these studies do provide general insights on HIV testing tendencies among HCWs. In a study of HIVST among HCWs in Ethiopia, it was reported that a higher proportion of nurses than doctors self-tested for HIV, but there was no difference by gender in the proportion of HCWs who reported self-testing [9]. Thus, there is limited information on factors associated with uptake of HIVST among HCWs, and our study has provided some insight on the characteristics of HCWs who find HIVST acceptable.

Several limitations to this study should be noted. First, the generalizability of the study may be limited given that the study population was not a random sample of HCWs at these facilities. Second, the cross-sectional nature of this study limits our ability to infer causality. Third, we do not have a record of those who refused the survey, thus we could not assess the bias due to refusal. However, based on the available records from the Ministry of Health of the staff size at these participating hospitals, we administered the survey to a median of $46 \%$ of the HCWs (interquartile range: 46-58 \%) in these hospitals. These records may have been outdated since at the time of our study, some of the personnel shown in the records were no longer working at the given hospitals. Another limitation is the lack of a true baseline assessment of sexual risk behaviors since the survey data was collected 1 month after the pre-HIVST information session. Therefore, the reported sexual behavior could have occurred after and been influenced by the pre-HIVST information session or the participants' self-test results. Future evaluations should assess sexual risk behaviors before and after HIVST. Additionally, all findings are based on self-report, which introduces biases, and the population may have under- or over-reported the use of HIVST kits due to fear about sharing the HIV selftest result or because HCWs were encouraged to use the HIVST kits. Future studies should incorporate procedures to validate self-reported use of HIVST kits. Lastly, given the fact that the HCWs who self-tested were not asked for outcomes of the HIV test, it was not possible to assess how many HCWs or their partners tested HIV positive and whether they sought confirmatory HIV testing and linkage to HIV care and treatment. Although HCWs were provided with contact details for the on-site coordinator in the survey, only one HCW reported to have made a phone call and the call was to seek clarification about the testing procedures. Hence this study was not able to examine the crucial matter of post-test counseling and linkage to care and treatment after self-testing. Future studies on HIVST should examine how to provide post-test counseling and link individuals to care and treatment after self-testing.

Despite these limitations, this study provides evidence of acceptability based on a real-world implementation as opposed to acceptability of HIVST based on a hypothetical 
question. The lessons learned from the HIVST intervention implementation among these HCWs highlight programmatic areas that need to be examined before expanding HIVST into other healthcare settings and the general population. The Kenya AIDS Indicator Survey (2012) showed that $72 \%$ of adults in Kenya have ever been tested for HIV and the country has implemented various strategies to increase access to HIV testing [16]. An innovative approach, such as HIVST, could help increase the uptake of HIV testing by reaching people who have not been accessing HIV test services due to fear of breach of confidentiality.

Although HIVST was included in the HTC policy in Kenya in 2006, HIVST has not yet been provided as a public health service due to the need for data to inform the design of implementation guidelines. Our study has made a contribution by demonstrating acceptability of HIVST and some characteristic of users among HCWs. However, during the international symposium on HIVST [10], participants from the Kenyan government indicated that more data was needed before promoting HIVST in the general population. To generate this data, the Kenya government, in collaboration with International Initiative for Impact Evaluation, initiated formative research activities around packaging and messaging, target population for HIVST, appropriate counseling strategies and linkage to care, appropriate distribution outlets, and potential for social harm. Findings from the formative research studies will be used to implement pilot projects on HIVST in Kenya before developing guidelines for wide implementation of HIVST.

\section{Recommendations}

The recommendations we provide below are specific to HIVST among HCWs in Kenya. However, given the paucity of data on this topic from other African countries, we believe these recommendations can be useful in the design of HIVST interventions for HCWs in other African countries.

1. The hurdles experienced in mobilizing HCWs to attend the pre-HIVST information sessions underscore the critical need to have buy-in of the management of the hospitals for the success of this program. Hospital management must address scheduling issues so that HCWs are given the opportunity to attend pre-HIVST information sessions and learn about HIVST.

2. The uptake of HIVST by partners of HCWs is encouraging given the known benefits of couples HTC [5] and that a large proportion of new infections result from heterosexual transmission within regular steady relationships. For future implementation, each HCW should be provided with a second HIVST kit to provide to the partner, and the self-test program should have a provision for $\mathrm{HCW}$ s to refer their partners to the on-site coordinator to train them and provide them with the HIVST kit.

3. Given that our study did not provide programmatic evidence on how to provide post-test counseling and care, future implementation of HIVST should examine innovative strategies of providing post-test counseling and linkage with care and support after HIVST such as the one in the South African study [17].

4. In addition, assessment of the HIV prevalence in the surrounding community may be needed to understand the context in which HCWs live and work.

5. Lastly, more research is needed on the sustainability and cost-effectiveness of implementing an unsupervised facility-based HIVST program.

\section{Conclusion}

In summary, unsupervised HIVST appears to be acceptable to surveyed HCWs, many of whom had self-tested previously. Furthermore, the majority of HCWs who attended the information session also used an HIVST kit, and many also reported HIVST was desirable to their sex partner(s). However, the greatest hurdle in the implementation of HIVST was getting HCWs to attend the pre-HIVST information sessions. The low attendance rate at the information sessions was likely due to job demands and staff not being available due to work shifts. In addition in some hospitals, the intervention team received insufficient support from hospital management when organizing the pre-HIVST information sessions, which could have affected the success in mobilizing HCWs to attend the sessions. Full support from hospital management may increase the use of HIV testing by HCWs in the future.

\section{References}

1. Tawfik L, Kinoti SN. The impact of HIV/AIDS on health systems and the health workforce in sub-saharan Africa. Geneva: World Health Organization; 2003.

2. Wilburn SQ, Eijkemans G. Preventing needlestick injuries among healthcare workers: a WHO-ICN collaboration. Int J Occup Environ Health. 2004;10(4):451-6.

3. US Agency for International Development. The health sector human resource crisis in Africa: an issues paper. Washington: USAID; 2003.

4. Mavedzenge SN, Baggaley R, Lo YR, Corbett L. HIV self-testing among health workers: a review of the literature and discussion of 
current practices, issues and options for increasing access to HIV testing in Sub-Saharan Africa Geneva, Switzerland: 2011.

5. National AIDS/STI Control Program (NASCOP). Guidelines for antiretroviral therapy in Kenya. 4th ed. Nairobi: National AIDS and STD Control Programme; 2011.

6. Taegtmeyer M, Suckling RM, Nguku PM, Meredith C, Kibaru J, Chakaya JM, et al. Working with risk: occupational safety issues among healthcare workers in Kenya. AIDS Care. 2008;20(3):304-10.

7. Makokha BB. Prevalence of accidental exposure to selected blood-borne pathogens and utilisation of post exposure prophylaxis among health workers in two Kenyan hospitals. 2012. Accessed 31 Oct 2013.

8. National AIDS/STI Control Program (NASCOP). Preparedness for HIV/AIDS service delivery: the Kenya health workers survey. National AIDS/STI Control Program (NASCOP); 2006.

9. Kebede B, Abate T, Mekonnen D. HIV self-testing practices among health care workers: feasibility and options for accelerating HIV testing services in Ethiopia. Pan Afr Med J. 2013;15:50.

10. World Health Organization (WHO). Report on the first international symposium on self-testing for HIV. Geneva: WHO;2013. http:// www.who.int/hiv/pub/vct/self_test/en/ Accessed 27 May 2014.

11. Kalibala S, Tun W, Muraah W, Cherutich P, Oweya E, Oluoch P. 'Knowing myself first': feasibility of self-testing among health workers in Kenya. Nairobi: Kenya Population Council; 2011.

12. Katz DA, Cassels SL, Stekler JD. Replacing clinic-based tests with home-use tests may increase HIV prevalence among Seattle men who have sex with men: evidence from a mathematical model. Sex Transm Dis. 2014;41(1):2-9.

13. World Bank. Kenya - HIV prevention response and modes of transmission analysis. Nairobi: World Bank; 2009. https://open knowledge.worldbank.org/handle/10986/3044 Accessed 13 May 2014.

14. Zungu LI, Sanni BA. Acceptance and uptake of voluntary HIV testing and among health care workers in a South Africa public hospital. S Afr Fam Pract. 2011;53(5):488-94.

15. Kirakoya-Samadoulougou F, Yaro S, Deccache A, Fao P, Defer MC, Meda N, et al. Voluntary HIV testing and risky sexual behaviours among health care workers: a survey in rural and urban Burkina Faso. BMC Public Health. 2013;13(1):540.

16. National AIDS/STI Control Program (NASCOP), Ministry of Health. Kenya AIDS Indicator Survey (KAIS), 2012preliminary report. Nairobi: NASCOP; 2013. http://nascop.or.ke/library/3d/ Preliminary\%20Report $\% 20$ for $\% 20$ Kenya $\% 20$ AIDS $\% 20$ indi cator\%20survey\%202012.pdf Accessed 13 May 2014.

17. Pant Pai N, Behlim T, Abrahams L, Vadnais C, Shivkumar S, Pillay S, et al. Will an unsupervised self-testing strategy for HIV work in health care workers of South Africa? A cross sectional pilot feasibility study. PLoS One. 2013;8(11):e79772. 